\title{
The Alarming Rate of Major Disruptive Events in Primary Care Practices in Oklahoma
}

James W. Mold, $M D, M P H^{1,2}$

Margaret Walsh, MS

Ann F. Chou, $\mathrm{PbD}, \mathrm{MPH}^{2}$

Juell B. Homco, MPH

'Oklahoma Medical Research Foundation, Oklahoma City, Oklahoma

${ }^{2}$ University of Oklahoma Health Sciences Center, Oklahoma City, Oklahoma

${ }^{3}$ University of Oklahoma School of Community Medicine, Tulsa, Oklahoma

\begin{abstract}
PURPOSE In primary care practices, sustainability of performance improvements and ability to deliver continuity of care to patients can be adversely affected by major disruptive events, such as relocations and changes in ownership, clinicians, and key staff. This study documented the rates of major disruptive events in a cohort of primary care practices in Oklahoma.
\end{abstract}

METHODS Practices were included if they had existed for 1 year before enrollment and remained in the project for at least 1 year after enrollment. Practice characteristics for 208 practices and major disruptive events during the preenrollment year were collected by survey. Postenrollment major disruptive events were prospectively collected by practice facilitators. We compiled frequency statistics and conducted bivariate analyses for each data set.

RESULTS Of 208 eligible practices, 81 (39\%) were clinician owned, and 51 (25\%) were health system owned. One hundred nine practices $(52 \%)$ were in nonmetropolitan counties. One hundred seventy-five major disruptive events occurred in $120(58 \%)$ practices during the preenrollment year, with 42 practices having experienced multiple events. During the first year of the project, 89 major disruptive events occurred in 67 (32\%) practices, with 20 practices experiencing multiple events. The major disruptive events reported most often during both periods were loss of personnel and implementation of electronic health records and billing systems. Practice size was associated with occurrence of these events.

CONCLUSIONS During a 2-year period, major disruptive events occurred at an alarming rate, adversely affecting quality improvement efforts. Most reported events involved losses of clinicians and staff. More research is needed to identify and address the root causes of these events.

Ann Fam Med 2018;16(Suppl 1)S52-S57. https://doi.org/10.1370/afm.2201.

\section{INTRODUCTION}

I $\mathrm{n}$ response to rising health care costs, the changing epidemiology of illnesses, and opportunities created by the advancement of information technologies, federal and state governments, insurance companies, and employers are applying pressure on primary care practices to transform the way they provide care. Common requirements involve implementation of electronic health records (EHRs), instituting population management and care management systems, and restructuring practice reimbursement from a fee-for-service model to value-based reimbursement models. These dramatic changes have created anxiety and uncertainty, which could be expected to result in some degree of organizational instability. ${ }^{1.7}$

Quality improvement directors and practice facilitators, who function as change agents helping primary care practices manage these challenges, routinely report that major disruptive events, such as relocations and changes in ownership, clinicians, and key staff, interfere with their efforts to effect change and sustain improvements. Surprisingly, the actual rate and impact of major disruptive events have rarely been documented or published. Although they can be difficult to track and report in a great number of practices over extended periods, there may also be reluctance 
on the part of implementation researchers to acknowledge the fragility of primary care practices and adverse effects of that fragility on transformation efforts.

As a part of the Healthy Hearts for Oklahoma study, a 3-year quality improvement project that enrolled primary care practices, we collected information about major disruptive events. Our objective was to document the number and type of disruptive events and to generate questions about impact and causation.

\section{METHODS}

Our study tracked 6 categories of major disruptive events occurring in a set of primary care practices in Oklahoma during a 2-year period. Data were collected by surveys of practice managers and staff, who reported retrospectively on major disruptive events that occurred within 1 year before study enrollment and obtained prospectively through reports entered by practice facilitators as they observed the workflow in their assigned practices.

All aspects of the project were approved by the University of Oklahoma Health Sciences Center Institutional Review Board, as well as the institutional review boards of Absentee Shawnee Tribe, Cherokee Nation, Chickasaw Nation, Choctaw Nation, Oklahoma City Area Indian Health Service, Integris Heath, and Ponca Tribe; all participants provided informed consent.

\section{Study Population}

In 2015 the Agency for Healthcare Research and Quality (AHRQ) launched EvidenceNOW: Advancing Heart Health in Primary Care, a multiyear pragmatic trial. EvidenceNOW is designed to generate information about the effectiveness of external quality improvement support in helping small and medium-size primary care practices use patient-centered outcomes research findings to improve the heart health of their patients. The project is also designed to determine the best ways to develop organizational capacity for ongoing practice improvement in primary care. Healthy Hearts for Oklahoma is 1 of 7 regional teams involved in the EvidenceNOW initiative.

During 2015 and 2016, Healthy Hearts for Oklahoma staff recruited and enrolled clinicians and staff in 263 practices distributed evenly across the state of Oklahoma. Enrolled practices were randomly assigned to 1 of 4 intervention waves in accordance with the project's stepped-wedge design. The quality improvement interventions included peer consultation provided by primary care clinicians trained by the National Resource Center for Academic Detailing, user-friendly summaries of current guidelines, on-site facilitation provided by certified practice facilitators, on-site information technology assistance provided by experts involved in Oklahoma's Health Information Technology Regional Extension Program, and monthly performance feedback.

We abstracted and analyzed data for the 208 practices that had existed for at least 1 year before enrollment and had participated for at least 1 year in the project.

\section{Data Collection}

A practice characteristics questionnaire was developed and administered online to all enrolled practices at baseline. The questionnaire was completed by the practice manager or clinician champion after the consent process and before the beginning of the intervention period. Questionnaires could be completed and directly entered into an electronic REDCap database (https://www.project-redcap.org). For those primary care practices that did not have REDCap access, practice facilitators administered paper questionnaires, then entered the responses into REDCap. The questionnaire elicited information about baseline practice characteristics, including size, ownership status, practice's readiness for change, and major disruptive events occurring during the 12 months before enrollment. Major disruptive event categories were established by the EvidenceNOW evaluation team.

An electronic practice record was developed to capture practice facilitator notes and observations. During the intervention period, practice facilitators entered information about major disruptive events directly into the electronic practice record system and posted facilitators and barriers to successfully working with their practices, as well as lessons learned. Although there was a menu of these disruptive events from which practice facilitators could check, they also had the opportunity to enter free text to describe the events in more detail. Practice personnel changes were also tracked on checklists submitted by facilitators to program managers, who maintained those changes in a project database. Data used for this report were abstracted from these data sources. When there were questions, the appropriate practice facilitator was contacted for clarification.

\section{Data Analysis}

The practice characteristics questionnaire did not specifically ask about the location of the practice (ie, urban or rural). To determine that information, practice addresses were geocoded using ArcGIS 10.3.1 (Environmental Systems Research Institute, Esri). Practices were then designated as metropolitan or nonmetropolitan based upon the Office of Management and Budget metropolitan county definition (US Census; Oklahoma 
During the first year postenrollment, 89 major disruptive events occurred in 67 (32\%) unique practices (Table 2), and 20 of those practices reported multiple disruptive events. Across the entire 2-year period (retrospective plus prospective), 137 (66\%) of the 208

Table 2. Major Disruptive Events Over 2 Years, Pre- and Postenrollment ( $\mathrm{N}=208$ Practices)

\begin{tabular}{|c|c|c|c|}
\hline \multirow[b]{2}{*}{ Event } & \multicolumn{2}{|c|}{ Practices Affected, No. (\%) } & \multirow{2}{*}{$\begin{array}{c}\text { No. of Unique } \\
\text { Practices } \\
\text { With MDEs }\end{array}$} \\
\hline & Preenrollment & Postenrollment & \\
\hline Lost 1 or more clinicians & $59(28)$ & $33(16)$ & 79 \\
\hline $\begin{array}{l}\text { Lost } 1 \text { or more office managers } \\
\text { or head nurses }\end{array}$ & $38(18)$ & $14(7)$ & 51 \\
\hline Moved to a new location & $9(4)$ & $8(4)$ & 17 \\
\hline $\begin{array}{l}\text { Purchased by or affiliated with } \\
\text { a larger organization }\end{array}$ & $1(<1)$ & $4(2)$ & 5 \\
\hline Implemented a new EHR & $33(16)$ & $15(7)$ & 43 \\
\hline New billing system & $35(17)$ & $15(7)$ & 42 \\
\hline $\begin{array}{l}\text { Total unique practices with } \\
\text { major disruptive events }\end{array}$ & $120(58)^{\mathrm{a}}$ & $67(32)^{b}$ & 137 \\
\hline
\end{tabular}

EHR = electronic health record; $M D E=$ major disruptive event.

Note: Data on preenrollment MDEs were collected via surveys of practice managers and staff who reported retrospectively on MDEs that occurred within 1 year before study enrollment. Postenrollment data on MDEs were obtained prospectively through reports entered by practice facilitators as they observed workflow in assigned practices. In all, 120 unique practices experienced at least $1 \mathrm{MDE}$ during preenrollment, and 67 unique practices reported at least 1 MDE postenrollment. During the 2-year period, 137 unique practices of the sample of 208 had at least 1 disruptive event.

a Forty-two practices experienced more than $1 \mathrm{MDE}$ during the preenrollment period.

${ }^{b}$ Twenty practices experienced more than 1 MDEs during the postenrollment period.

\section{Figure 1. Number of practices by total number of major disruptive} events.

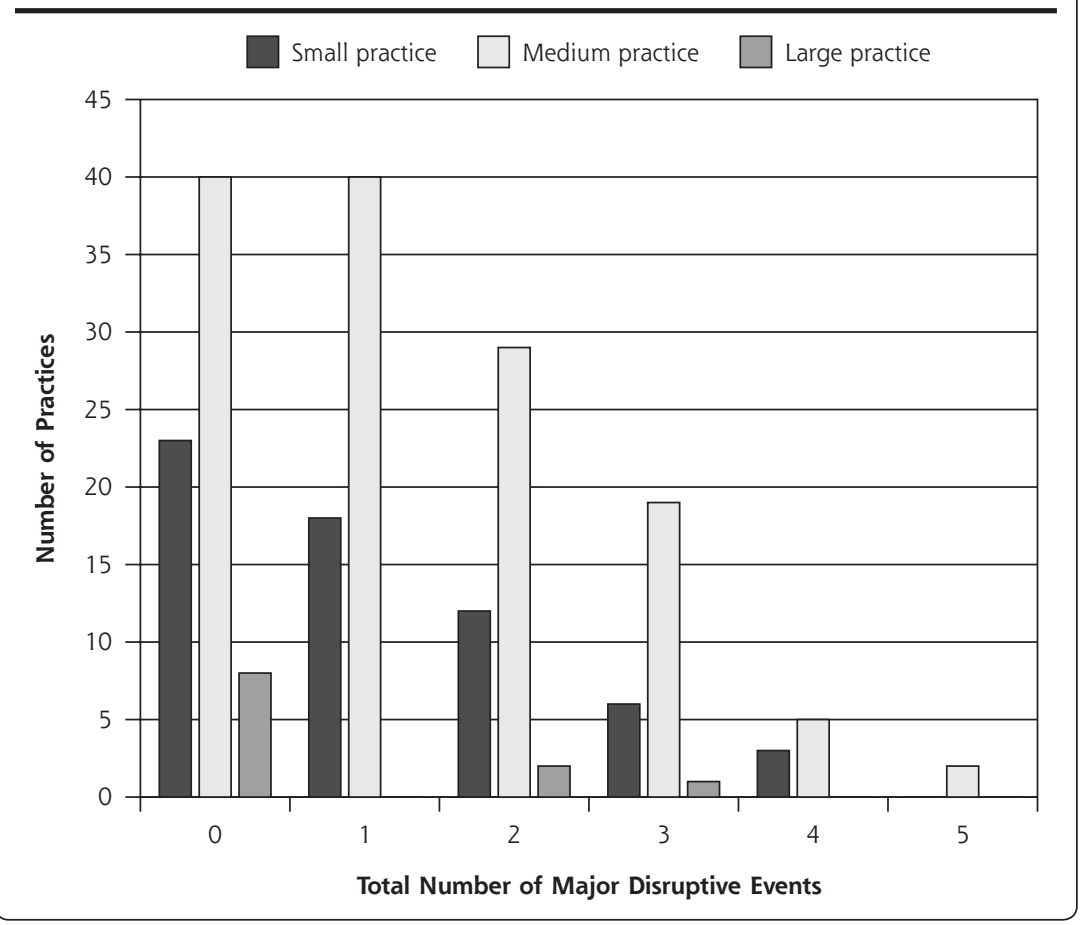

practices experienced at least 1 major disruptive event Comparing the second year of the study period with the first, major disruptive events related to technology or human resources occurred less frequently by more than $50 \%$, movement of practice locations remained the same, and the number of practices that were purchased by or became affiliated with a health care system increased.

Figure 1 displays the number of major disruptive events as a function of practice size. About $63 \%$ of small primary care practices, $70 \%$ of medium-size practices (2 to 10 clinicians), but only $27 \%$ of large practices ( $>10$ clinicians) reported at least 1 major disruptive event. Medium-size practices were somewhat more likely to experience at least 1 disruptive event than solo and larger practices $\left(\chi^{2}=8.75 ; P=.01\right)$ (Figure 1). Fifty percent of small and medium-size primary care practices experienced 1 to 2 disruptive events. More medium-size practices $(19 \%)$, however, reported having 3 or more major disruptive events compared with $15 \%$ of small practices. Only 1 large practice reported 3 such disruptive events. No statistically significant associations were found between practice location, ownership, or number of clinicians and occurrence of at least 1 disruptive event during the 2-year period.

Although we specifically excluded practices that dropped out of the study during the first year, disruptive events had a major impact on the capacity of those remaining practices in implementing and continuing their quality improvement activities, which was anecdotally supported by practice facilitators' entries in the electronic practice records. This practice facilitator's notes documented the effects of personnel change, the most commonly occurring disruptive event, on the practice's ability to achieve its quality improvement goals: 
We had a clinic that was part of a health system. To recruit the clinic to $\mathrm{H} 2 \mathrm{O}$ [Healthy Hearts for Oklahoma], we had to meet several times with different levels of the organization: medical director, clinic manager, clinician, etc. Ultimately, they did decide to participate. At different times during the project, the organization has lost both the medical director and the clinic manager. After each person was replaced, the new employee made us freeze the $\mathrm{H} 2 \mathrm{O}$ project until they could get their feet under them and decide whether the clinic could continue to participate in the project. Shutting down the project multiple times during the year of intervention not only affected the momentum of the staff participants, but it affected the PDSA [plan, do, study, act] cycles such that we couldn't tell for sure whether the changes were having the desired effect, or whether other variables were responsible.

Another practice facilitator noted the disruptions caused by technology change:

One of my clinics is switching from a family-owned practice to being bought out by a corporate company to become the local hospital clinic. The provider does not touch a computer. The nurses do all of the documentation and scribing for the provider. These nurses have been with this provider since the beginning, and these transitions of going from paper to electronic have not been their best moments. I have heard countless stories of them wanting to go back to paper because electronic takes so much more time. They are very old school in this clinic and do not like change. They have gotten used to this system and they [are] finally used to workflow and enjoy using it. Now they are being forced to change EHRs again. This has brought out many struggles and frustration among the staff. They didn't want the change the first time, and now they are having to change again.

These entries from the cadre of practice facilitators at Healthy Hearts for Oklahoma lend further insights into the possible impact of major disruptive events on the quality improvement efforts of primary care practices.

\section{DISCUSSION}

Our finding that two-thirds of the practices in our sample experienced at least 1 major disruptive event during a 2-year period should be alarming to everyone involved in practice transformation efforts. The fact that the practices in our sample had willingly enrolled in a quality improvement initiative and participated for at least 1 year suggests the actual rate of major disruptive events in primary care practices may be even higher.

We identified only 1 comparable study, published in 1997, involving primary care practices in Minnesota. ${ }^{8}$ In that study, Magnan et al reported an even higher 1-year rate of ownership changes (64\%), changes in practice manager or medical director
(45\%), personnel changes (59\%), and EHR implementation $(45 \%)$ than we have.

The most frequent major disruptive events involved loss of key personnel. Personnel loss, both clinical and managerial, decreases organizational resources (eg, organizational competencies, and human capital) from which organizations may draw support in times of uncertainty. ${ }^{9}$ Inadequate staffing inhibits the ability of the practice to participate in improvements as well as routine training activities. ${ }^{10}$ The loss of clinical staff can be particularly problematic, because clinicians often provide both leadership and cognitive support for quality improvement. ${ }^{11}$

The rate of major disruptive events did not vary across ownership types or geography (metropolitan vs rural). Even though it makes mathematical sense that practices with more clinicians and staff would be at greater risk for personnel changes, medium-size practices in our study reported greater staff changes or loss than did the solo and large practices. Solo practices are less complex and possibly more cohesive, resulting in higher levels of clinician and staff allegiance, thus avoiding certain types of major disruptive events. Mediumsize practices, by contrast, may lack the resources and buffers of larger practices and the cohesiveness of small practices, making them most susceptible.

Improvements in care processes are only beneficial if they can be sustained. Although sustainability requires more study, it is likely that stability of clinicians, staff, and record systems are important, because those are the main sources of institutional memory. In the face of major disruptive events, clinician and staff training programs are likely to be essential. Factors accounting for personnel loss need to be studied to identify mitigating strategies.

\section{Limitations}

Our study, which was strengthened by a relatively large number of practices, statewide representation, and prospective in-practice observations by practice facilitators, had several limitations. Our study sample included practices from only 1 state, which may limit generalizability. Project selection criteria excluded practices with more than 10 full-time-equivalent primary care clinicians, practices that did not take care of a substantial number of adults, and practices with no EHR. Although the total number of practices was reasonably large, the sample did not provide sufficient power to detect small to moderate subgroup effects.

We did not record the loss of nonadministrative nurses and medical assistants, who are often responsible for making new management systems work. We were also unable to capture turnover rates, which would have allowed us to control for exposure (eg, size 
of practice). Finally, there was no way to verify the accuracy of the retrospective survey data. For example, practices might have remembered and reported some major disruptive events that had occurred more than a year before enrollment. The accuracy of prospective data was dependent upon practice facilitators' reporting either via electronic practice records or enrollment checklists. Practice facilitators were only in each practice for a few hours every 2 weeks. If a practice facilitator was not made aware that a major disruptive event had occurred, they could not report it.

Major disruptive events deserve much greater attention than they have received, as they could impede primary care delivery and practice transformation efforts. In particular, more information is needed to determine the causes of major disruptive events and the effects of the various disruptive events on both quality improvement engagement and sustainability of improvements and on the quality and cost of care.

To read or post commentaries in response to this article, see it online at http://www.AnnFamMed.org/content/16/Suppl_1/S52.

Key words: primary care; quality improvement; continuity of care; electronic health records; disruptions; turmoil; implementation

Submitted June 13, 2017; submitted, revised, November 16, 2017; accepted November 28, 2017.

Funding support: Publication of this article was supported by The Agency for Healthcare Research and Quality (AHRQ) through contract No. HHSA290201200019I, and grant No. R18 HS023919.

Disclaimer: This work represents the opinions of the authors and should not be interpreted as official positions of the Agency for Healthcare Research and Quality or the US Department of Health and Human Services.
Acknowledgments: We thank the practice facilitators who expanded great efforts to collect these data and to work with these practices. We are grateful to the practices that participated in this study.

Supplementary materials: Available at http://www.AnnFamMed. org/content/16/Suppl_1/S52/suppl/DC1/.

\section{References}

1. Zucker LG. Institutional theories of organization. Annu Rev Sociol. 1987; $13: 443-464$

2. Meyer JW, Rowan B. Institutional organizations: Formal structure as myth and ceremony. Am J Sociol. 1977;83(2):340-363.

3. DiMaggio PJ, Powell WW. The iron cage revisited: Institutional isomorphism and collective rationality in organizational fields. Am Sociol Rev. 1983;48(2):147-160.

4. Rogers EM. The innovation-decision process. In: Rogers E, ed. Diffusion of Innovations. $5^{\text {th }}$ ed. New York, NY: Free Press; 2003.

5. Levitt B, March JG. Organizational learning. Annu Rev Sociol. 1988; 14:319-338.

6. Huber, GP. Organizational learning: The contributing processes and the literatures. Organ Sci. 1991;2:88-115.

7. Tolbert $P$, Zucker L. Institutional sources of change in the formal structure of organizations: The diffusion of civil service reform, 1880-1935. Adm Sci Q. 1983;28:22-39.

8. Magnan S, Solberg LI, Giles K, Kottke TE, Wheeler JW. Primary care, process improvement, and turmoil. J Ambul Care Manage. 1997;20(4):32-38.

9. Scott WR. Organizations: Rational, Natural, and Open Systems. Upper Saddle River, NJ: Prentice Hall; 2003.

10. Zinn J, Flood AB. Commentary: Slack resources in health care organizations - fat to be trimmed or muscle to be exercised? Health Serv Res. 2009;44(3):812-820.

11. Geonnotti K, Taylor EF, Peikes D, Schottenfeld L, Burak H, McNellis R, and Genevero J. Engaging Primary Care Practices in Quality Improvement: Strategies for Practice Faciliators. AHRQ Publication No. 15-0015-EF. Rockville, MD: Agency for Healthcare Research and Quality; 2015.

12. Bodenheimer T, Pham HH. Primary care: current problems and proposed solutions. Health Aff (Millwood). 2010;29(5):799-805. 